\title{
Softwarelokalisierung - ein neues Arbeitsfeld für Übersetzerinnen und Übersetzer
}

\author{
Klaus-Dirk Schmitz \\ Fachhochschule Köln
}

Internationalization and localization are keywords in our present global economy. The Development and marketing of products and services are successful only if they can be adapted to requirements of the target market in a fast and easy way. The adaptation of software products that are linguistically, culturally, legally and technically appropriate for a regional market is called software localization.

This paper gives an overview of the field of software localization. After some general definitions of important concepts, the types of "texts" that have to be localized and the types of software tools that are used for localization are described. Special linguistic and non-linguistic aspects relevant and specific to localization in general are discussed and explained using examples.

\section{Einleitung}

Internationalisierung und Lokalisierung zählen heute zu den Schlüsselbegriffen einer globalen Marktwirtschaft. Will sich ein Unternehmen erfolgreich auf dem Weltmarkt behaupten, müssen Produkte und Dienstleistungen so entwickelt werden, dass sie schnell und einfach an die Anforderungen des Zielmarktes angepasst werden können. Die Erstellung von sprachlich, kulturell und technisch an einen bestimmten lokalen Markt angepassten Produkten und Dienstleistungen nennt man heute Lokalisierung.

Die enorme technische Entwicklung im Bereich des Personalcomputers und die starke Verbreitung dieses Rechnerkonzepts seit Beginn der 80er Jahre haben ebenso wie die Orientierung der meist in den USA beheimateten Softwareentwickler auf nicht-englischsprachige Märkte seit Anfang der 90er Jahre dazu geführt, dass Softwareprodukte heute weltweit vermarktet werden. Gesetze zur Produkthaftung und EU-Richtlinien sowie die starke Verbreitung des Internet haben ebenfalls ihren Anteil daran, dass die Anpassung von Softwareprodukten an regionale Märkte, d.h. die Softwarelokalisierung, zu einem riesigen Wirtschaftsfaktor in einem stark wachsenden Markt und zu einem neuen Arbeitsfeld für Übersetzerinnen und Übersetzer geworden ist.

Diese neuen Anforderungen des Marktes müssen natürlich auch in den translatorischen Studiengängen berücksichtigt werden sowie in spezifischen Weiterbildungsangeboten der Hochschulen und Berufsverbänden einfließen. Was dabei neu ist und in welchen Bereichen das traditionelle Curriculum überarbeitet bzw. ergänzt werden muss, soll dieser Beitrag erläutern. 


\section{Wichtige Begriffsklärungen und Begriffsabgrenzungen}

Auch wenn in der Einleitung intuitiv deutlich geworden ist, womit sich die Lokalisierung beschäftigt, so ersetzt dies nicht die genaue Definition des Begriffes selbst, die Erläuterung einiger verwandter Begriffe sowie die Abgrenzung der Begriffe untereinander.

\subsection{Globalisierung (G11N)}

Globalisierung umfasst alle Aktivitäten eines Unternehmens im Hinblick auf die Vermarktung eines Produktes oder einer Dienstleistung außerhalb des nationalen Marktes. Ziel der Globalisierung ist ein erfolgreiches Marketing in vielen unterschiedlichen regionalen Märkten unter besonderer Berücksichtigung der dort geltenden technischen, wirtschaftlichen und gesetzlichen Aspekte. Globalisierung ist immer im Kontext der betriebswirtschaftlichen und kaufmännischen Unternehmensführung zu sehen; deshalb sind hiervon vorwiegend die Marketing-Abteilungen der Unternehmen betroffen.

\subsection{Internationalisierung (I18N)}

Unter Internationalisierung verstehen wir die (technische) Entwicklung von Produkten und Dienstleistungen im Hinblick auf eine leichte Anpassung an andere Märkte, d.h. an andere (technische) Konventionen, kulturelle Eigenheiten, gesetzliche Auflagen und Sprachen. Ziel der Internationalisierung ist es, Produkte so zu entwickeln, dass Produktversionen für (neue) regionale Märkte schnell und mit möglichst geringem Aufwand erstellt werden können; eine Neukonzeption, Neuentwicklung oder Neuprogrammierung sollte in jedem Fall vermieden werden. Aus diesem Grund sind alle Bemühungen zur Internationalisierung der Produktentwicklung und der daran beteiligten Abteilungen eines Unternehmens zuzuordnen. Eine enge Kooperation mit den Stellen, die die Produkte und Dienstleistungen an die lokalen Märkte anpassen, ist unbedingt anzustreben.

\subsection{Lokalisierung (L10N)}

Den Prozess der Anpassung eines Produktes oder einer Dienstleistung an einen regionalen Markt, d.h. an einen unterschiedlichen Sprach- und Kulturraum, bezeichnet man als Lokalisierung. Ziel der Lokalisierung ist es, technisch, sprachlich und kulturell angemessene Produktversionen für die jeweiligen Märkte zu erstellen, indem man textliches Material übersetzt und nicht-textliches Material anpasst. Deswegen gehört die Lokalisierung in der Regel zu den Aufgabenbereichen von Übersetzern, technischen Autoren und Lokalisierungsfachleuten. 


\subsection{Zusammenwirken von Internationalisierung und Lokalisierung}

Internationalisierung und Lokalisierung umfassen den gesamten Prozess der Entwicklung von Produkten und Dienstleistungen für lokale Märkte. Der Aufwand für die Internationalisierung ist nur einmal bei der Produktentwicklung zu erbringen, der Aufwand für die Lokalisierung jedoch mehrmals für die Produktanpassung an den jeweiligen neuen Markt. Betreibt deshalb ein Unternehmen einen hohen Aufwand bei der Internationalisierung von Produkten, verringert sich der Aufwand für die Lokalisierung dieser Produkte entsprechend. Je mehr aus der Lokalisierung herausgenommen und in die Internationalisierung hineingesteckt werden kann, desto einfacher, schneller und billiger wird der gesamte Produkt-Entwicklungsprozess für alle Märkte.

\subsection{Abgrenzung von Lokalisierung und Übersetzen}

Unter Übersetzen verstehen wir das Übertragen eines schriftlichen Textes der Ausgangssprache in einen schriftlichen Text der Zielsprache. Bei einem heute üblichen funktionalistischen Verständnis von Übersetzen schließt dies auch die Anpassung des Zieltextes an die kulturellen und technischen Gegebenheiten des Zielmarktes ein.

Eine Translation ist nicht die Transkodierung von Wörtern oder Sätzen aus einer Sprache in eine andere, sondern eine komplexe Handlung, in der jemand unter neuen funktionalen und kulturellen und sprachlichen Bedingungen in einer neuen Situation über einen Text (Ausgangssachverhalt) berichtet, indem er ihn auch formal möglichst nachahmt. (Vermeer 1986:33)

Besteht ein zu lokalisierendes Produkt hauptsächlich aus Text, sind sich demnach Übersetzen und Lokalisieren sehr nahe. Auch die Benutzeroberfläche von Software, d.h. Menü-Optionen, Schaltflächen, Auswahlfelder, Fehlermeldungen und Online-Hilfe, kann als eine besondere Art Text gesehen werden, der an die Anforderungen des Zielmarktes angepasst werden muss. Bei der Softwarelokalisierung kommen aber durch bestimmte wirtschaftliche, organisatorische und technische Rahmenbedingungen Faktoren hinzu, die normalerweise nicht zum Übersetzen im engeren Sinn gerechnet werden können.

Translation is only one of the activities in localization; in addition to translation, a localization project includes many other tasks such as project management, software engineering, testing, and desktop publishing. (Esselink 2000:4)

\section{Was wird lokalisiert?}

Versucht man die bei der Lokalisierung von Software anzupassenden "Texte" zu analysieren und systematisieren, so ergibt sich folgende Übersicht: 


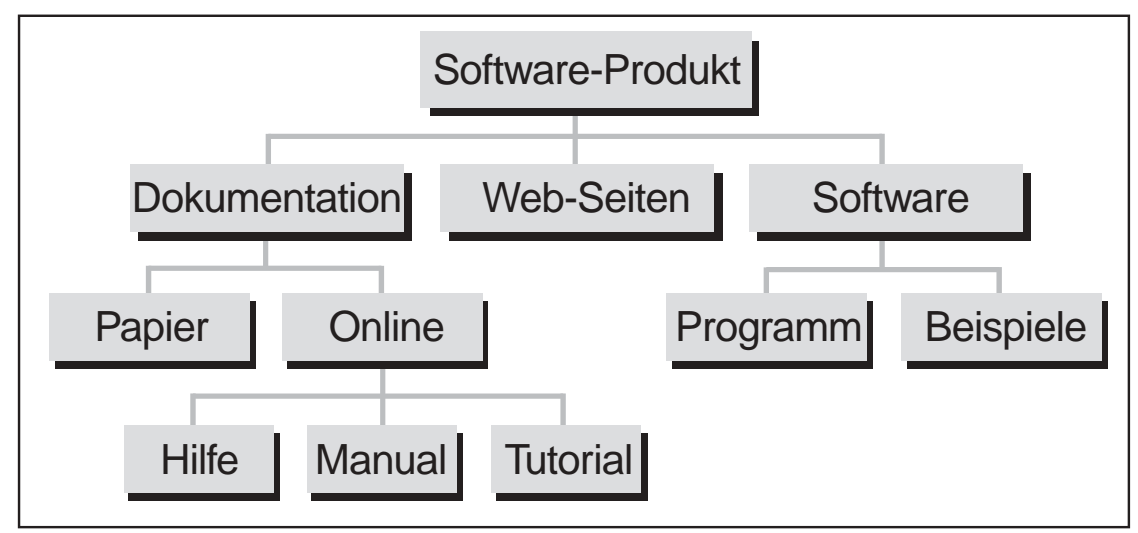

Abb. 1: Übersicht über zu lokalisierende Objekte

\subsection{Lokalisierung der Dokumentation}

Normalerweise werden Softwareprodukte mit einer umfangreichen Dokumentation ausgeliefert, die dem Benutzer hilft, das Programm zu installieren, die Bedienung des Programms zu erlernen und spezielle Funktionen bei Bedarf nachzuschlagen. Handbücher und Installationsanweisungen werden häufig ergänzt um Werbematerial, Garantiekarten, Registrierungskarten oder Verpackungsaufschriften. All diese Teile der Produktdokumentation müssen an die regionalen Märkte angepasst werden.

In der Regel liegt der ausgangssprachliche Text in elektronischer Form vor und wird meist in dem DTP- oder Textverarbeitungsprogramm, in dem er verfasst wurde, mit den zielsprachlichen Entsprechungen überschrieben, um die Formatierungsinformationen zu erhalten. In einigen dieser Programme werden Inhaltsverzeichnisse, Indizes und Querverweise auf andere Textstellen automatisch verwaltet bzw. generiert, in anderen muss beim Lokalisieren darauf geachtet werden, dass der Wortlaut der entsprechenden Textstellen (z.B. Überschriften) mit dem der Verzeichnisse übereinstimmt und dass die Seitenzahlen korrekt sind. Ebenso müssen Grafiken und Bildschirmmasken in der Dokumentation lokalisiert und angepasst oder evtl. neu aus der lokalen Version der Software übernommen werden.

\subsection{Lokalisierung der Online-Hilfe}

Besondere Formen der Dokumentation sind Online-Hilfen und Lernprogramme. Sie unterscheiden sich von den Handbüchern insbesondere durch das so genannte Hypertext-Prinzip, d.h. die Möglichkeit, durch einen Mausklick schnell von einer Stelle zu einer anderen zu wechseln (oder auch von einem bestimmten Dialogfeld in der Software aus die zugehörige Hilfeseite aufzurufen). Dies erfordert eine Codierung der entsprechenden Textstellen in der Hilfe bzw. im Lernprogramm. Die Texte, die der Benutzer sieht, werden 
in der Regel aus einem Format erzeugt, in dem solche Codierungen erstellt werden können. Dieses zugrundeliegende Format, in dem auch die Übersetzung erfolgen muss, ist bei Online-Hilfen unter Windows in der Regel das Dateiformat RTF (in jüngster Zeit auch HTML). Bei der Übersetzung der Hilfetexte ist insbesondere $\mathrm{zu}$ beachten, dass die Codierung unverändert bleibt, also keine Zeichenfolge geändert wird, die dem Hilfeprogramm das Ziel einer Verknüpfung (engl. link) angibt oder die zum Aufbau des Suchindexes benötigt wird. Ähnlich verhält es sich bei den Lernprogrammen. Beim Lokalisieren von Hilfen und Lernprogrammen ist ebenso wie bei der Dokumentation ein Einbinden der lokalisierten Grafiken und Bildschirmmasken erforderlich.

\subsection{Lokalisierung der Software}

Auch die Software selbst, d.h. die Benutzeroberfläche, muss lokalisiert werden. Die Texte der Benutzeroberfläche (Menübefehle, Listenfelder, Dialogfeld-Elemente, Schaltflächen etc.) sind normalerweise nicht ohne weiteres zugänglich. Es gibt spezielle Hilfsprogramme, mit denen der zu bearbeitende Text vom Programmcode isoliert und für die Übersetzung bereitgestellt werden kann. Nach Beendigung des Übersetzungsvorgangs ist das Kompilieren der übersetzten Quelldateien in das ausführbare Programm sowie das ausführliche Testen der einzelnen Funktionen in der lokalisierten Version der Software erforderlich.

\subsection{Lokalisierung von Web-Seiten}

In letzter Zeit wird auch die Übersetzung und Anpassung von Web-Seiten dem Bereich der Softwarelokalisierung zugerechnet, da diese ähnlich wie die Online-Hilfe in einem speziellen Format (HTML) vorliegen und Seiten untereinander mit Verknüpfungen verbunden sind. Bei der Lokalisierung von Web-Seiten, z.B. mit Hilfe spezieller HTML-Editoren, ist darauf zu achten, dass die HTML-Codierung der Web-Seiten-Gestaltung nicht zerstört wird; allerdings können gerade hierbei auch kulturspezifische Anpassungen erforderlich werden.

\section{Welche Werkzeuge braucht man bei der Softwarelokalisierung?}

Die Lokalisierung von Software und der dazugehörigen Dokumentation ist ohne den Einsatz entsprechender elektronischer Werkzeuge nicht (effizient) möglich. Neben Programmen zur Textverarbeitung, zum DTP, zur Bearbeitung von Grafiken und zur Terminologieverwaltung werden bei der Softwarelokalisierung häufig Translation-Memory-Programme eingesetzt, mit denen die Übersetzungen gespeichert werden. Dies ist insbesondere wegen des repetitiven Charakters der Texte und wegen der häufig anfallenden 
Lokalisierung von aktualisierten Softwareupdates sinnvoll. Für die Lokalisierung der Software selbst stehen ebenfalls spezielle Programme zur Verfügung.

\subsection{Textverarbeitungsprogramme und Editoren}

Bei der Lokalisierung der Dokumentation erfolgt die Übersetzung in der Regel in dem DTP- oder Textverarbeitungsprogramm (z.B. Adobe FrameMaker, Adobe PageMaker, Interleaf oder Microsoft Word), in dem der Originaltext verfasst wurde; eine Umwandlung des Dateiformats zum Zweck der Bearbeitung in Translation-Memory-Programmen ist jedoch auch üblich. Der Originaltext wird meist überschrieben, um die Formatierungsinformationen beizubehalten. Das unbeabsichtigte Löschen oder Verändern von Formatinformationen oder sonstigen Steuerzeichen, z.B. bei Hilfedateien oder Web-Seiten, kann durch die Benutzung spezieller Editoren verhindert werden. Für das Bearbeiten bzw. Übersetzen von Bildschirmmasken und Grafiken werden die Programme verwendet, in denen die jeweiligen Originale erstellt wurden oder die das entsprechende Dateiformat unterstützen. Bildschirmmasken werden häufig als BMP- oder als TIF-Dateien gespeichert und in das DTP-Programm eingebunden. Für die Bearbeitung von Grafiken werden gängige Programme wie Adobe Illustrator oder CorelDraw verwendet.

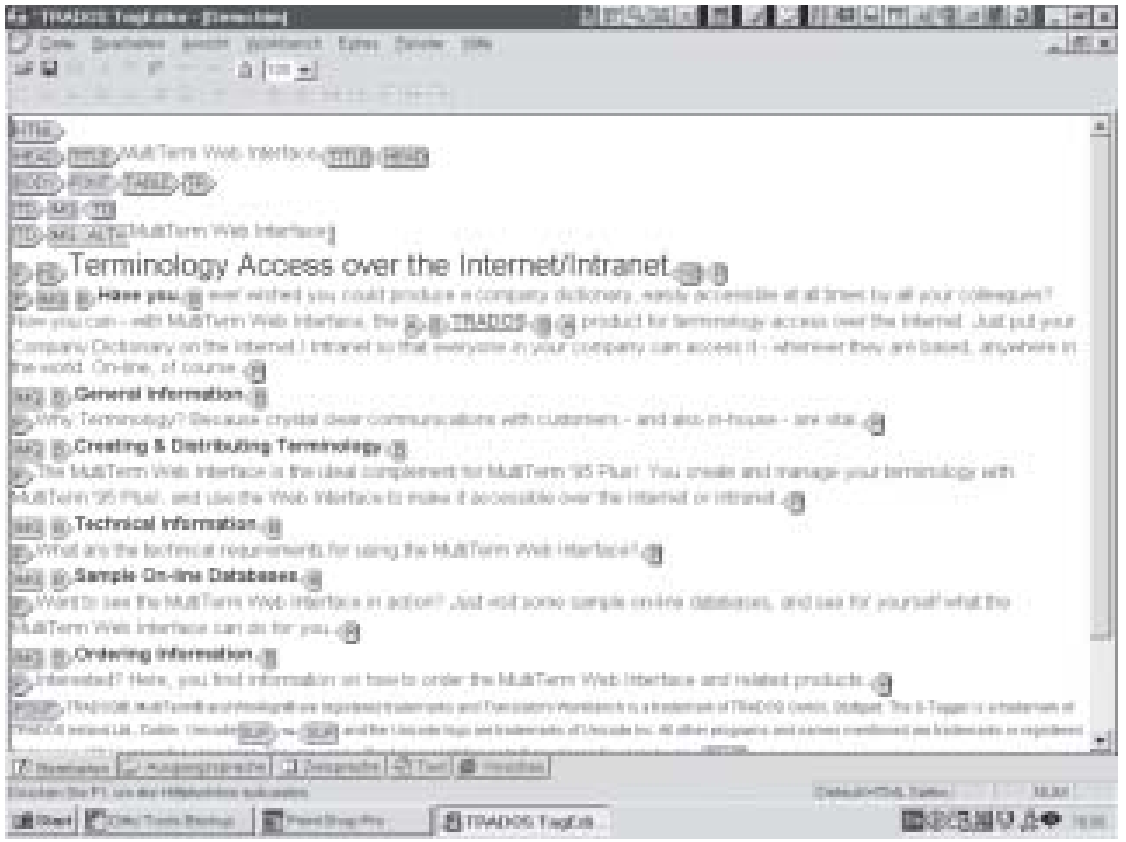

Abb. 2: Beispiel für einen Editor: Trados TagEditor 


\subsection{Terminologieverwaltungsprogramme}

Terminologieverwaltungsprogramme erlauben es, Fachwörter und fachsprachliche Wendungen in mehreren Sprachen effizient zu verwalten und auf diese während des Lokalisierungsprozesses schnell zuzugreifen. Dadurch wird nicht nur die Suche nach unbekannten Termini beschleunigt, sondern auch die Verwendung einer einheitlichen Terminologie unterstützt, was insbesondere bei größeren Textmengen und bei mehreren an einem Projekt beteiligten Übersetzern notwendig ist. Je nach Konzeption des Terminologieverwaltungsprogramms arbeitet man benennungs- oder begriffsorientiert, können zwei oder mehrere Sprachen verwaltet werden und stehen unterschiedlich viele Datenkategorien zur Dokumentation der Fachtermini zur Verfügung. Für die Anwendung im Bereich der Softwarelokalisierung ist es besonders wichtig, dass das Terminologieverwaltungsprogramm dem Prinzip der Benennungsautonomie folgt, d.h. dass mehrere unterschiedliche Benennungen zum gleichen Begriff mit Angaben zum Projekt, Produkt oder Kunden, dem sie zuzuordnen sind, versehen werden können. Es kommt nämlich sehr häufig vor, dass der gleiche Softwareentwickler unterschiedliche Terminologie für unterschiedliche Produktfamilien, ja sogar für verschiedene Versionen der gleichen Software verwendet. Terminologieverwaltungsprogramme sollten zusammen mit den anderen Werkzeugen des Lokalisierers benutzbar sein und mit ihnen zusammenwirken, z.B. mit der Textverarbeitung, den DTP-Programmen und den Translation-MemoryProgrammen.

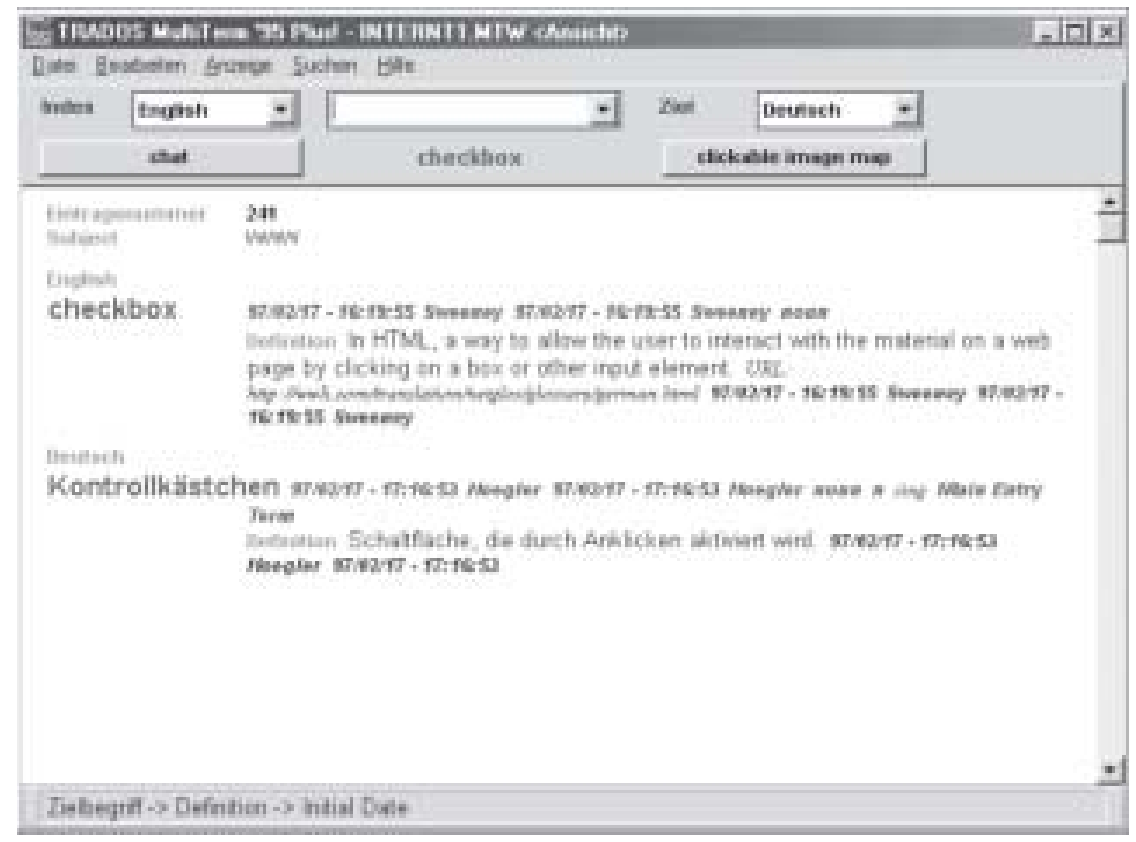

Abb. 3: Beispiel für ein Terminologieverwaltungsprogramm: Trados MultiTerm 


\subsection{Translation-Memory-Programme}

Translation-Memory-Programme (Übersetzungsspeicher) sind Werkzeuge, bei denen übersetzte Textsegmente (in der Regel Sätze) zusammen mit den entsprechenden ausgangssprachlichen Segmenten in einer Datenbank gespeichert werden. Diese Datenbank kann im Netzwerk abgelegt und mehreren Übersetzern gleichzeitig zur Verfügung gestellt werden. Trifft ein Übersetzer auf einen Satz, der mit einem bereits übersetzten Satz ganz oder teilweise übereinstimmt, wird dessen Übersetzung als Vorschlag angezeigt und kann direkt oder nach Modifikation in das zielsprachliche Dokument übernommen werden. Hierdurch wird den Übersetzern zum einen die Mühe erspart, den gleichen Satz mehrmals zu übersetzen, und zum anderen wird eine Einheitlichkeit im Sprachstil und in der verwendeten Terminologie gewährleistet, was insbesondere von Vorteil ist, wenn mehrere Übersetzer an einem Projekt arbeiten. Die auf dem Markt verfügbaren Translation-Memory-Programme arbeiten nach unterschiedlichen Prinzipien, sind aber meistens in eine Umgebung eingebunden, die zusätzlich noch eine Terminologieverwaltungskomponente und einen Übersetzungseditor enthält.

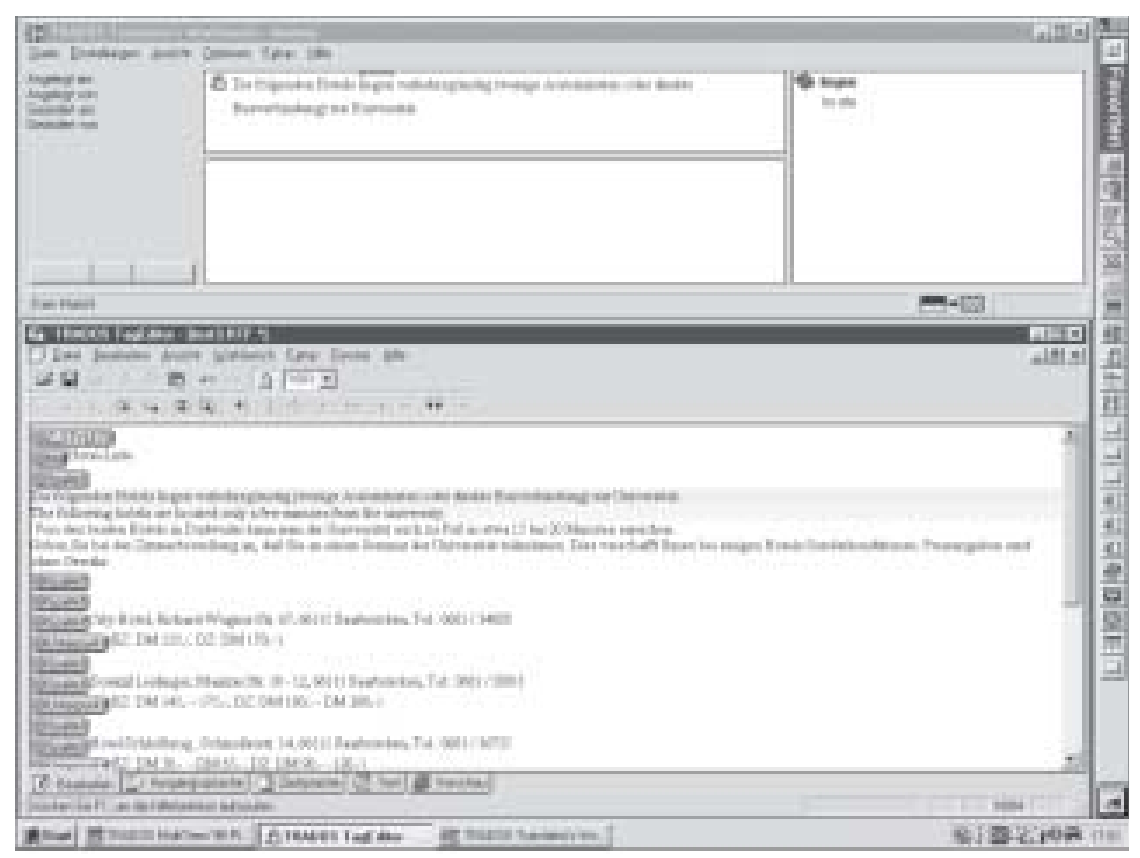

Abb. 4: Beispiel für ein Translation-Memory-Programm: Trados Workbench mit TagEditor

\subsection{Spezielle Lokalisierungsprogramme}

Zur Lokalisierung von Softwaredateien gibt es Programme, mit denen der zu bearbeitende Text "WYSIWYG" (What you see is what you get) übersetzt 
werden kann. Das bedeutet, dass z.B. der Text in einem englischen Dialogfeld direkt mit der deutschen Entsprechung überschrieben wird. Dies ermöglicht ein kontextbezogenes Übersetzen und erleichtert das erforderliche Anpassen der Textfelder an die Länge des Zieltextes. Für das Testen der Online-Hilfe werden ebenfalls spezielle Programme eingesetzt, mit denen die einzelnen Funktionen und Verknüpfungen innerhalb der Hilfe im Anschluss an den Übersetzungsprozess systematisch überprüft werden können.

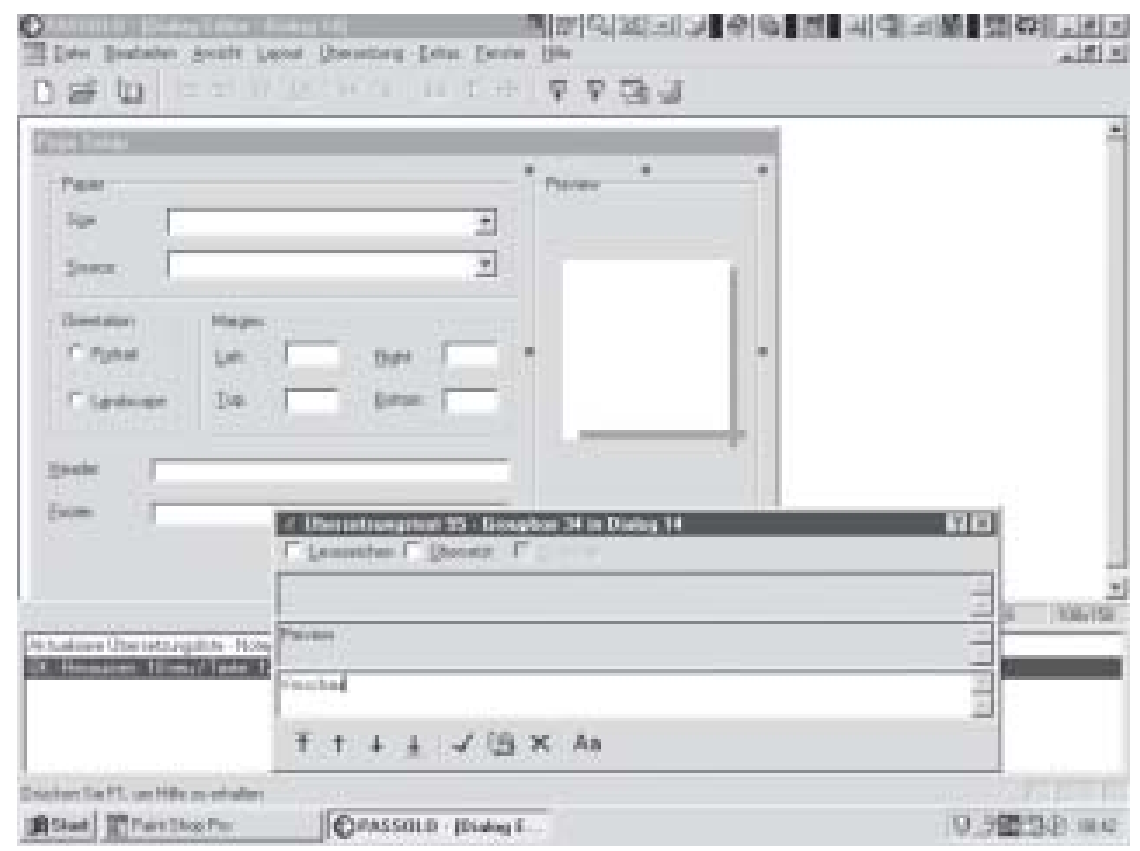

Abb. 5: Beispiel für ein Lokalisierungsprogramm: Passolo

\section{Besondere Aspekte der Softwarelokalisierung}

Im Folgenden sollen besondere Aspekte diskutiert werden, die bei der Softwarelokalisierung, aber auch schon bei der Entwicklung von Software im Sinne der Internationalisierung, zu berücksichtigen sind.

\subsection{Terminologie}

Die Auswahl und Festlegung sowie die konsistente und korrekte Verwendung von Fachwörtern (Terminologie) sind Grundvoraussetzungen für eine erfolgreiche Lokalisierung. Die Nutzer eines bestimmten Zielmarktes müssen und wollen in ihrer jeweiligen Muttersprache angesprochen werden, wobei je nach Fachlichkeitsgrad des Produktes oder der Dienstleistung der Anteil der fachspezifischen Terminologie unterschiedlich groß sein kann. Oft betreffen zu lokalisierende Softwareprodukte innovative Fachgebiete 
und Themen, bei denen die zu verwendende Terminologie in der Zielsprache (noch) nicht existiert oder etabliert ist. Deshalb muss die Terminologie erst geprägt werden, bevor mit dem Lokalisierungsprozess begonnen werden kann; entsprechende Regeln zur Bildung von Fachwörtern (z.B. Motiviertheit von Termini) sowie zur Sprachpflege und Sprachplanung (z.B. Vermeidung von unnötigen Anglizismen) sind unbedingt zu berücksichtigen.

Da es sich bei Softwarelokalisierungsprojekten meist um größere Vorhaben (mit engen Zeitvorgaben) handelt, an denen mehrere Mitarbeiter beteiligt sind, ist die Klärung der ausgangssprachlichen Terminologie und die Festlegung der zielsprachlichen Äquivalente vor dem Beginn der Lokalisierung nicht nur aus Effizienz-, sondern auch aus Konsistenzgesichtspunkten notwendig. Sonst würden viele Mitarbeiter den gleichen Ausgangsbegriff recherchieren und im Extremfall $\mathrm{zu}$ unterschiedlichen zielsprachlichen Entsprechungen kommen. Hinzu kommt, dass eine durchgängig einheitliche Terminologie in allen zu lokalisierenden Produkten verwendet werden muss. Für den Bereich der Softwarelokalisierung bedeutet dies, dass auf der Programmoberfläche, in der Online-Hilfe, in der schriftlichen Dokumentation und auf den Produkt-Webseiten die gleichen Benennungen für die gleichen Begriffe verwendet werden müssen.

In vielen Lokalisierungsprojekten wird eine einheitliche und abgesicherte Terminologie vorgegeben, die unbedingt eingehalten werden muss. Hierbei handelt es sich entweder um eine festgelegte unternehmensspezifische Terminologie, die im Rahmen der Corporate Identity als Unternehmenssprache (Corporate Language) definiert ist, oder Gesetze und Richtlinien schreiben die Verwendung einer bestimmten Terminologie (z.B. in Warnhinweisen oder Garantiebedingungen) vor. Ebenso kann die Einbettung eines bestimmten Produktes in eine festgelegte Umgebung die Verwendung der spezifischen Terminologie dieser Umgebung erfordern; wird etwa eine bestimmte Software lokalisiert, die auf Windows-Rechnern eingesetzt werden soll, so ist die etablierte Windows-Terminologie zu berücksichtigen.

\subsection{Symbole}

Entwickler versuchen gerne im Rahmen der Internationalisierung, einige der zu lokalisierenden sprachlichen Elemente durch die Verwendung von Symbolen oder Abbildungen zu ersetzen, um den Aufwand für die Lokalisierung gering zu halten. Auch wenn der Ansatz im Prinzip nicht falsch ist, so muss doch festgestellt werden, dass viele Symbole nicht nur kulturspezifisch, sondern oft auch sprachspezifisch sind.

Symbole werden wegen des Bekanntheitsgrades oft aus anderen, meist universelleren Lebensbereichen, die natürlich kulturgeprägt sind, übernommen, um etwas Abstraktes mit einem konkreten Beispiel zu verdeutlichen. Besonders schwierig wird es, wenn Symbole sprachliche Anspielungen ausnutzen, indem sie über Homonyme (oder gar Homophone) auf einen anderen Begriff verweisen. 
Die folgenden Beispiele aus der Softwarelokalisierung mögen dies verdeutlichen:

\begin{tabular}{|l|l|}
\hline & $\begin{array}{l}\text { Englische Benennung: trash } \\
\text { Deutsche Benennung: Papierkorb } \\
\text { Problem: Abbildung passt nicht zur Benennung }\end{array}$ \\
\hline & $\begin{array}{l}\text { Englische Benennung: clipboard } \\
\text { Deutsche Benennung: Zwischenablage } \\
\text { Problem: Abbildung passt nicht zur Benennung }\end{array}$ \\
\hline & $\begin{array}{l}\text { Englische Benennung: home } \\
\text { Deutsche Benennung: Anfang oder Startseite } \\
\text { Problem: Abbildung passt nicht zur Benennung }\end{array}$ \\
\hline
\end{tabular}

Abb. 6: Beispiele für Lokalisierungsprobleme bei Symbolen

Da Symbole bei der Software meist in die Programmoberfläche fest integriert sind, können sie bei der Lokalisierung nicht oder nur mit viel Aufwand verändert und angepasst werden. Ähnliches gilt für Symbole, die bei technischen Produkten (Drucker, Maschinen, Auto) fest auf Tasten und Schaltern angebracht sind.

\subsection{Abbildungen, Grafiken und Fotos}

Dass Abbildungen, Grafiken und Fotos, die kulturspezifische Situationen (z.B. religiöse Symbole und Gebäude, Aussehen und Kleidung von Personen, Landschaften und Städte) zeigen, bei einer Lokalisierung durch entsprechende andere ersetzt werden sollten, versteht sich von selbst. Ebenso muss bei Abbildungen, Grafiken und Fotos, die sich auf Produkte beziehen, die bei der Lokalisierung für einen anderen Markt verändert wurden (z.B. Kraftfahrzeug mit Links-/Rechtslenkung, Programmoberflächen von lokalisierter Software), eine entsprechende neue Darstellung erzeugt und in die lokalisierte Version der Dokumentation eingebunden werden. 
Neben den Inhalten können auch Darstellungskonventionen bei Abbildungen und Grafiken kulturspezifisch variieren. Auch die Anordnung und das Layout von Abbildungen und Grafiken muss bei der Lokalisierung angepasst werden, wenn in bestimmten lokalen Märkten andere Konventionen für die technische Dokumentation (z.B. bei Software-Handbüchern) gelten.

Als ein Fehler bei der Internationalisierung kann es bezeichnet werden, wenn sprachliche Elemente in der Abbildung oder Grafik enthalten sind. Es kann sehr mühsam sein, mit speziellen Bildbearbeitungsprogrammen die ausgangssprachlichen Textelemente zu löschen und durch zielsprachliche Elemente zu ersetzen. Oft muss deswegen die gesamte Grafik neu erstellt werden, auch wenn die Abbildung selbst wegen ihrer KulturNeutralität beibehalten werden könnte.

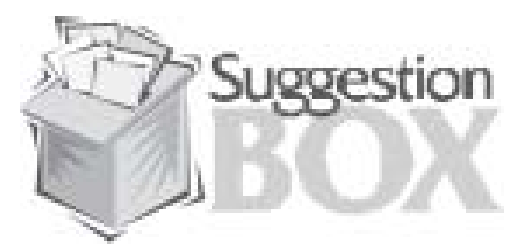

Abb. 7: Zu lokalisierendes Textelement in Grafik eingebunden

\subsection{Farben}

Da Farben in unterschiedlichen Kulturen eine unterschiedliche Bedeutung haben, müssen diese bei einer Lokalisierung gegebenenfalls verändert werden. So wird etwa Tod und Trauer im westeuropäischen Kulturraum mit der Farbe Schwarz in Verbindung gebracht, während hierfür in Japan und China eher die Farbe Weiß benutzt wird. Da auch Farben von Warnhinweisen zwischen den Kulturen variieren, wird die Signalwirkung bestimmter Farben lokal unterschiedlich interpretiert. Es ist auch nicht ungewöhnlich, dass die Farben der Landesflagge in einer bestimmten Nation bevorzugt, z.B. bei der Gestaltung von Internetseiten, eingesetzt werden. (Abb. 8) 


\begin{tabular}{|l|l|l|l|l|}
\hline \multicolumn{5}{|c|}{ Kulturspezifische Farbbedeutungen } \\
\hline & Westeuropa & Japan & China & arab. Länder \\
\hline rot & Gefahr & $\begin{array}{l}\text { Aggression } \\
\text { Gefahr }\end{array}$ & $\begin{array}{l}\text { Freude } \\
\text { festliche } \\
\text { Stimmung }\end{array}$ & \\
\hline gelb & $\begin{array}{l}\text { Vorsicht } \\
\text { Feigheit }\end{array}$ & $\begin{array}{l}\text { Wurde } \\
\text { Adel }\end{array}$ & & $\begin{array}{l}\text { Gluck } \\
\text { Wohlstand }\end{array}$ \\
\hline grün & $\begin{array}{l}\text { sicher } \\
\text { Neid }\end{array}$ & $\begin{array}{l}\text { Zukunft } \\
\text { Jugend } \\
\text { Energie }\end{array}$ & $\begin{array}{l}\text { Fruchtbarkeit } \\
\text { Starke }\end{array}$ \\
\hline blau & $\begin{array}{l}\text { Männlichkeit } \\
\text { Kalte } \\
\text { Autorität }\end{array}$ & $\begin{array}{l}\text { Reinheit } \\
\text { Tugend }\end{array}$ & $\begin{array}{l}\text { Tod } \\
\text { Trauer }\end{array}$ & $\begin{array}{l}\text { Tod } \\
\text { Trauer }\end{array}$ \\
\hline weiß & $\begin{array}{l}\text { Tod } \\
\text { Trauer }\end{array}$ & & & Wertrauen \\
\hline schwarz & Waheit \\
\hline
\end{tabular}

Abb. 8: Kulturspezifik von Farben: www.kommdesign.delfakten/seite2.htm

Eine weitere detaillierte Übersicht zur Farbverwendung findet sich unter: www.farbenundleben.de/kulturspezifische_Farbbeutungen.htm.

\subsection{Zeichensätze}

Eine Grundvoraussetzung für die Lokalisierung der sprachlichen Elemente eines Produktes ist es, dass die Sprache des Zielmarktes durch den entsprechenden Zeichensatz adäquat repräsentiert und verarbeitet werden kann. Hierzu gehört z.B. auch die korrekte Sortierung der Wörter einer Sprache.

Vor allem bei Software-Produkten war die korrekte und vernünftige Behandlung nicht-englischer Zeichensätze früher problematisch. Durch die Erweiterung des 7-Bit-Zeichensatzes auf den erweiterten ASCII-Zeichensatz konnten zumindest die westeuropäischen lateinischen Zeichensätze eindeutig dargestellt werden; Kyrillisch und Griechisch konnten meist nur alternativ und unter Aufgabe anderer Zeichen benutzt werden.

Mit der Einführung des Windows-Betriebssystems und der Verfügbarkeit lokalisierter Versionen für den asiatischen Markt standen Codepages für unterschiedliche Zeichensätze zur Verfügung. Allerdings zeigte sich immer wieder, dass das Ansprechen der relevanten Codepage nicht einfach war und nicht immer die erwarteten Ergebnisse zeigte. 
Heute ist durch die Einführung des UNICODE-Standards (16-Bit oder 32-Bit) eine einheitliche und eindeutige Basis gelegt, die eine vernünftige Behandlung der Zeichensätze (fast) aller Sprachen der Welt ermöglicht. Sind Software-Produkte unter Verwendung von UNICODE entwickelt und internationalisiert, sollte die Lokalisierung bezüglich der Zeichensätze unproblematisch sein.

\subsection{Schriften und Typographie}

Schriftarten und Schriftfamilien werden in Kulturen unterschiedlich häufig und für unterschiedliche Textsorten und Textteile verwendet. So kann man beispielsweise in englischsprachigen Anleitungen eher Schriften mit Serifen (z.B. Times Roman), in deutschen Anleitungen eher serifenlose Schriften (z.B. Helvetica) finden. Ebenso verwendet das Englische häufiger Versalien (reine Großschreibung) für Überschriften und hervorzuhebende Textpassagen als das Deutsche, da der Informationsverlust durch Großbuchstaben beim Lesen dieser Textteile wegen der im Englischen kaum vorhandenen Groß-/ Kleinschreibung sehr gering ist.

Auch für Textgliederungselemente existieren kulturspezifisch unterschiedliche Konventionen; im Deutschen wird eher ein Spiegelstrich ("-"), im Englischen ein Punkt (bullet “•”) verwendet. Ähnliches gilt für Aufzählungen mit Nummerierungen, aber auch für Anführungszeichen und Apostrophe (Beispiele finden sich in Schmitz \& Wahle 2000:101ff). Es zeigt sich allerdings, dass sich in diesem Bereich die kulturspezifische Verwendung von Schriften und Typographie nach und nach immer mehr an die amerikanischen Konventionen anpasst, da für die Erstellung von Texten Software mit amerikanischem Ursprung verwendet wird.

\subsection{Sprache und Stil}

Über die einzelsprachspezifischen Möglichkeiten des Satzbaus und des Stils gibt es kulturelle Konventionen, die bei der Lokalisierung unbedingt zu beachten sind. So wird im (US-) Englischen oft salopp geschrieben, der Leser wird persönlich angesprochen, es werden einfache Verben benutzt, Textteile wiederholt und Termini undifferenziert benutzt, während im Deutschen bevorzugt fachspezifische Verben benutzt werden, formal sauber geschrieben wird, nicht wiederholt wird, möglichst keine Synonyme verwendet werden und eher unpersönlich, passivisch formuliert wird.

Auch kulturspezifischen Konventionen und lokalen gesetzlichen Auflagen bei standardisierten Textpassagen (z.B. bei Sicherheitshinweisen) sind bei der Lokalisierung Aufmerksamkeit zu schenken.

Deutsch: Vorsicht! Vor dem Öffnen Netzstecker ziehen! 


\section{Englisch: DO NOT REMOVE OUTER COVER}

To prevent electric shock, do not remove cover.

No user serviceable parts inside.

Refer servicing to qualified service personnel.

Man beachte in dem aus Schmitt (1999:184) übernommenen Beispiel neben den Standardformulierungen auch Unterschiede in der Typographie (Versalien) und in der Verwendung des Ausrufezeichens.

Besonders bei der Formulierung von Überschriften gibt es unterschiedliche Konventionen. Die folgenden Beispiele der Lokalisierung von Überschriften aus EDV-Dokumentationen vom Englischen ins Deutsche (aus Schmitz \& Wahle 2000:118f) mögen dies verdeutlichen.

Englisch: To install the software

Deutsch: So installieren Sie die Software nicht: Um die Software zu installieren nicht: Wie Sie die Software installieren nicht: Installieren der Software

Englisch: What you need

Deutsch: Erforderliche Ausstattung / Komponenten nicht: Was Sie benötigen

Typisch für die englische Sprache und im Bereich der Softwarelokalisierung besonders häufig zu finden sind "Mammutkomposita", die natürlich nicht Wort-für-Wort ins Deutsche übertragen werden dürfen. Die korrekte Auflösung der Komposita im Deutschen erfordert meist eine sehr genaue Kenntnis der Sachlage.

\section{Englisch: Network Printing Services Setup}

Deutsch: richtig: Einrichtung der Druckdienste im Netzwerk oder: Einrichtung der Netzwerkdruckdienste nicht: Netzwerkdruckdienste-Einrichtung

Englisch: the Windows 98 operating system

Deutsch: das Betriebssystem Windows 98 nicht: das Windows 98-Betriebssystem

Eine Besonderheit ergibt sich bei der Lokalisierung von Elementen der Benutzeroberfläche (Menüs, Dialogfelder, Befehle ...) von Software. Neben der stilistischen Forderung, im Deutschen zuerst die Art des Elements und dann das Element zu nennen statt Komposita zu verwenden (z.B. "das Menü Datei”, statt "das Datei-Menü”), ergibt sich eine andere, durch die im Deutschen übliche Großschreibung erzwungene Forderung. Während in englischen EDV-Texten Elemente der Benutzeroberfläche im Fließtext durch Großschreibung hervorgehoben werden und dadurch auffallen, müssen im Deutschen, wo auch andere Substantive im Satz groß geschrieben 
werden, diese Elemente durch andere Textauszeichnungen (z.B. Fettschrift, Anführungszeichen) kenntlich gemacht werden.

Englisch: Open the Edit menu

Deutsch: Öffnen Sie das Menü Bearbeiten

Öffnen Sie das Menü „Bearbeiten“

nicht: das Bearbeitenmenü / das Bearbeiten-Menü

In jedem Fall sind bei der Lokalisierung sprachlicher Elemente die Stilrichtlinien (style guides) des Auftraggebers zu beachten, sofern solche vorhanden sind. Darüber hinaus gilt, dass vor allem eine Kontinuität im sprachlichen Stil unbedingt einzuhalten ist, wobei einfach strukturierte Sätze bevorzugt und Bandwurmsätze vermieden werden sollten. Auch wenn dies häufig im Ausgangstext nicht der Fall ist, müssen in der lokalisierten Fassung klare Bezüge sichergestellt und die logische Reihenfolge der Informationen beibehalten bzw. hergestellt werden. Weitere Empfehlungen und Beispiele finden sich u.a. in Schmitt 1999 und Schmitz \& Wahle 2000.

\subsection{Formate}

Dass in unterschiedlichen Kulturen andere Maßangaben für Entfernungen, Gewichte oder Füllmengen verwendet werden, ist allgemein bekannt. Diese müssen ebenso wie Datumsformate, Papierformate und Währungsangaben bei der Lokalisierung angepasst werden. Ob diese Angaben exakt umgerechnet werden müssen oder nicht, hängt von der Aussagefunktion der Information im Original ab. So kann beispielsweise die Angabe "250 USDollar" zu "250 Euro" oder zu einem Euro-Betrag genau nach dem Tageskurs lokalisiert werden. Im folgenden Beispiel (aus Schmitz \& Wahle 2000:127) wäre eine exakte Umrechnung der Maßangaben inadäquat:

Englisch: Allow at least 6 inches of clearance on all sides and 12 inches above the printer.

$(6$ inches $=15.24 \mathrm{~cm})$

Deutsch: An allen Seiten des Druckers muss ein Mindestabstand von $15 \mathrm{~cm}$ und oberhalb des Druckers ein Abstand von $30 \mathrm{~cm}$ eingehalten werden.

Vor allem im Bereich der Softwarelokalisierung, aber auch bei der Beschriftung von Tasten und Schildern bei Maschinen, muss berücksichtigt werden, dass die Textlänge je nach Sprache variiert. Während sich in der schriftlichen Dokumentation der Seitenumfang, die Seitenzahl der einzelnen Informationen und der Bezug darauf im Inhaltsverzeichnis und Index verändert, muss bei der Lokalisierung der Programmoberfläche gegebenenfalls die Größe der Schaltflächen und Dialogboxen angepasst werden. 


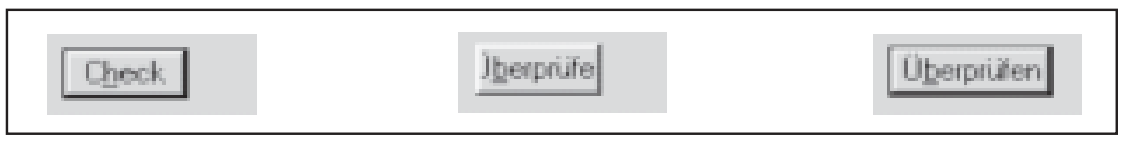

Abb. 9: Anpassung der Größe von Schaltflächen

\subsection{Beispiele}

Die Anpassung von Beispielen kann einen recht großen Anteil bei der Lokalisierung eines Produktes oder einer Dienstleistung ausmachen. Bei einer Textverarbeitungssoftware müssen beispielsweise alle Standardbriefvorlagen, bei einer Visitenkartensoftware alle Muster-Visitenkarten an die Konventionen des Zielmarktes sowie typische Namen, Orte und Straßen angepasst werden.

Das folgende Beispiel aus Schmitz \& Wahle (2000:128) soll verdeutlichen, dass auch bei einer beispielorientierten Beschreibung einer Benutzeraktion das Beispiel nach der Lokalisierung immer noch "funktionieren" muss.

Englisch: Click the Environment folder, and then SHIFT+click the Sport folder. Press DEL to delete all folders.

Deutsch: Klicken Sie auf den Ordner Umwelt und dann bei gedrückter UMSCHALTTASTE auf den Ordner Sport. Drücken Sie ENTF, um alle Ordner zu löschen.

(korrekt übersetzt, aber nicht funktionstüchtiges Beispiel)

Deutsch: Klicken Sie auf den Ordner Freizeit und dann bei gedrückter UMSCHALTTASTE auf den Ordner Umwelt. Drücken Sie ENTF, um alle Ordner zu löschen.

(korrekt lokalisiert, da das Beispiel jetzt funktioniert)

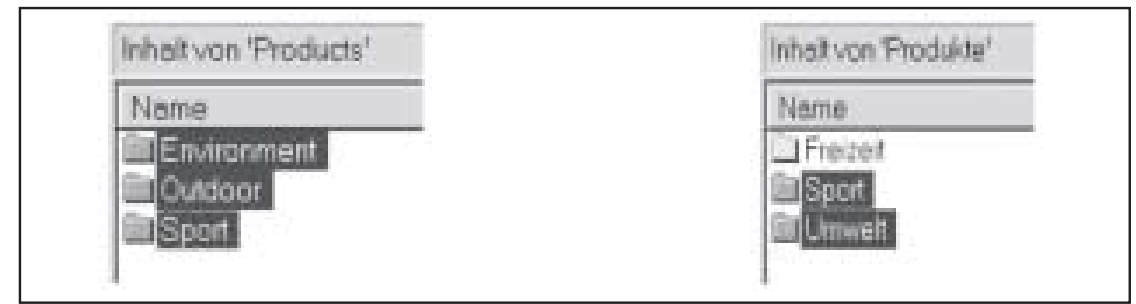

Abb. 10: Lokalisierung einer beispielorientierten Benutzeraktion

\section{Ausblick}

Die Lokalisierungsaktivitäten werden meist durch große, weltweit operierende Lokalisierungsunternehmen mit Sitz in den USA oder in Europa (vorwiegend in Irland) koordiniert. In den einzelnen Zielländern arbeiten auf Lokalisierung spezialisierte Übersetzungsunternehmen und viele Freiberuf- 
ler an Lokalisierungsprojekten. Der Markt ist so umfangreich und wächst so schnell, dass ein dringender Bedarf an Lokalisierungsexperten besteht. Dies ist eine große Herausforderung und Chance für Übersetzer, die sich im Bereich der Softwarelokalisierung spezialisiert haben oder dies zurzeit tun.

Dies ist aber gleichzeitig auch eine Herausforderung an die Ausbildungsinstitute, ihre Absolventen in diesen Zweig der Berufspraxis einzuführen und in Kooperation mit den Berufsverbänden entsprechende Weiterbildungsmaßnahmen zu entwickeln und anzubieten. Dabei geht es nicht nur um eine Erweiterung der Sachfachausbildung im Bereich der Informationstechnologie und um die Vorbereitung auf den Umgang mit den erforderlichen, spezialisierten Werkzeugen zur Lokalisierung; auch terminologische und computerlinguistische Arbeitsmethoden müssen vermittelt werden. Im Bereich der Übersetzungswissenschaft ist sicherlich eine Orientierung in Richtung der funktionalen Übersetzungstheorie für die Ausbildung im Bereich der Softwarelokalisierung hilfreich. Aus Sicht der Fachsprachenforschung und Linguistik allgemein ist die Untersuchung der bei der Softwarelokalisierung typischerweise auftretenden Textsorten interessant; während die schriftliche Dokumentation in Form von Handbüchern und Anleitungen eher bekannte linguistische Phänomene aufweist, weisen "Texte" wie Webseiten, Online-Hilfen und vor allem Bedienoberflächen von Programmen eher unübliche linguistische Strukturen auf, die bisher wenig untersucht wurden. Theoretische und praktische Anleitungen zur Analyse und Übersetzung dieser Text- und Satzstrukturen müssen erarbeitet werden und in die Ausbildung einfließen.

\section{Bibliographie}

Esselink, Bert (2000). A Practical Guide to Localization. Amsterdam/Philadelphia: John Benjamins.

Freigang, Karl-Heinz (1999). "Softwarelokalisierung.” Technische Kommunikation 2(1999), 23-26.

Freigang, Karl-Heinz \& Klaus-Dirk Schmitz (2002). "Softwarelokalisierung als Aufgabe für Übersetzer.” Joanna Best \& Sylvia Kalina (Hrsg.) (2002). Übersetzen und Dolmetschen. Eine Orientierungshilfe. Tübingen: Francke, 242-248.

Schmitt, Peter A. (1999). Translation und Technik. Studien zur Translation. Bd.6. Tübingen: Stauffenburg.

Schmitz, Klaus-Dirk (2001). ,Softwarelokalisierung.” Felix Mayer (Hrsg.) (2001). Dolmetschen \& Übersetzen - Der Beruf im Europa des 21. Jahrhunderts. Freiburg: freigang,mauro+reinke, 173-181.

Schmitz, Klaus-Dirk (2002). "Werkzeuge zur Lokalisierung von Software: Catalyst 4.0 und Passolo 3.5." Technische Kommunikation 3(2002), 24-28.

Schmitz, Klaus-Dirk \& Kirsten Wahle (Hrsg.) (2000). Softwarelokalisierung. Tübingen: Stauffenburg.

Vermeer, Hans J. (1986). “Übersetzen als kultureller Transfer." Mary Snell-Hornby (Hrsg.) (1986). Übersetzungswissenschaft - Eine Neuorientierung. Tübingen: Francke, 30-53.

Wright, Sue Ellen \& Gerhard Budin (Hrsg.) (2001). Handbook of Terminology Management. Volume II. Amsterdam/Philadelphia: John Benjamins. 